\title{
Positivity of the Weights of Extended Gauss-Legendre Quadrature Rules
}

\section{By Giovanni Monegato*}

Abstract. We show that the weights of extended Gauss-Legendre quadrature rules are all positive.

1. Introduction. We consider extended Gauss-Legendre quadrature formulas, i.e., integration rules of the type

$$
\int_{-1}^{1} f(x) d x=\sum_{i=1}^{n} A_{i}^{(n)} f\left(\xi_{i}^{(n)}\right)+\sum_{j=1}^{n+1} B_{j}^{(n)} f\left(x_{j}^{(n)}\right)+R_{n}(f)
$$

where $\xi_{i}^{(n)}, i=1, \ldots, n$, are the zeros of the $n$th degree Legendre polynomial $P_{n}(x)$, while the nodes $x_{j}^{(n)}, j=1,2, \ldots, n+1$, and the weights $A_{i}^{(n)}, B_{j}^{(n)}$ are chosen so that (1) has degree of exactness $p=3 n+1\left(3 n+2\right.$ if $n$ is odd), i.e., $R_{n}(f)=0$ whenever $f$ is a polynomial of degree up to $p$. If we denote by $E_{n+1}(x)$ the polynomial of degree $n+1$, whose zeros are the abscissas $x_{j}^{(n)}, j=1,2, \ldots, n+1$, then $E_{n+1}(x)$ has to satisfy the following orthogonality relation

$$
\int_{-1}^{1} P_{n}(x) E_{n+1}(x) x^{k} d x=0, \quad k=0,1, \ldots, n .
$$

Szegö [4] has studied $E_{n+1}(x)$ in a different context and gives some very interesting results. For instance, he proves that the nodes $x_{j}^{(n)}$ are in $(-1,1)$ and interlace with the zeros of $P_{n}(x)$.

Formulas for the computation of the weights $A_{i}^{(n)}$ and $B_{j}^{(n)}$ are given in [2], [3]. In [2] it is shown that the $B_{j}^{(n)}$ 's are positive; however, nothing has been said about the sign of $A_{i}^{(n)}$. In this note we show that the weights $A_{i}^{(n)}$ are also positive.

2. Positivity of $A_{i}^{(n)}$. We consider the Legendre function of second kind

$$
Q_{n}(x)=\frac{1}{2} \int_{-1}^{1} \frac{P_{n}(t)}{x-t} d t, \quad n \geqslant 1,
$$

defined for any $x$ in the complex plane cut along the segment $[-1,1]$; we introduce the function

$$
\bar{Q}_{n}(x)=\frac{1}{2} \lim _{\epsilon \rightarrow+0}\left[Q_{n}(x+i \epsilon)+Q_{n}(x-i \epsilon)\right],
$$

which is analytic on $(-1,1)$. It is known $[5, \mathrm{p} .78]$ that 
(4)

$$
\lim _{\epsilon \rightarrow+0}\left[Q_{n}(x+i \epsilon)-Q_{n}(x-i \epsilon)\right]=-i \pi P_{n}(x), \quad-1<x<1
$$

From (2), (3) and (4), and recalling Lebesgue's convergence theorem, it then follows that at the zeros $\xi_{i}^{(n)}, i=1, \ldots, n$, of $P_{n}(x)$ we have

$$
\bar{Q}_{n}\left(\xi_{i}^{(n)}\right)=\frac{1}{2} \int_{-1}^{1} \frac{P_{n}(t)}{\xi_{i}^{(n)}-t} d t
$$

Let now

$$
E_{n+1}(\cos \phi)=\lambda_{0} \cos (n+1) \phi+\lambda_{1} \cos (n-1) \phi+\cdots+ \begin{cases}\lambda_{n / 2} \cos \phi, & n \text { even, } \\ 1 / 2 \lambda_{(n+1) / 2}, & n \text { odd }\end{cases}
$$

and

$$
e_{n+1}(\phi)=\lambda_{0} \sin (n+1) \phi+\lambda_{1} \sin (n-1) \phi+\cdots+ \begin{cases}\lambda_{n / 2} \sin \phi, & n \text { even } \\ 0, & n \text { odd }\end{cases}
$$

where $x=\cos \phi, 0<\phi<\pi$, and, as known [4], $\lambda_{0}=(2 n+1) ! /\left(2^{2 n}(n !)^{2}\right)$. Then, Szegö in his paper $[4$, p. 507] gives the following inequality

$$
\bar{Q}_{n}(\cos \phi) E_{n+1}(\cos \phi)+\frac{\pi}{2} P_{n}(\cos \phi) e_{n+1}(\phi)>1, \quad 0<\phi<\pi,
$$

which implies that at the nodes $\xi_{i}^{(n)}$

$$
\left|E_{n+1}\left(\xi_{i}^{(n)}\right)\right|>\left|\bar{Q}_{n}\left(\xi_{i}^{(n)}\right)\right|^{-1}, \quad i=1, \ldots, n .
$$

We are now ready to prove the following

THEOREM. The weights $A_{i}^{(n)}$ and $B_{j}^{(n)}$ of the extended Gauss-Legendre rules are always positive.

Proof. The positivity of $B_{j}^{(n)}$ has already been proved in [2]. In that paper, the following expression for the weights $A_{i}^{(n)}$ has also been given

$$
A_{i}^{(n)}=H_{i}^{(n)}-\frac{h_{n}}{k_{n}\left|P_{n}^{\prime}\left(\xi_{i}^{(n)}\right)\right|\left|q_{n+1}\left(\xi_{i}^{(n)}\right)\right|}, \quad i=1, \ldots, n,
$$

where $H_{i}^{(n)}=2\left|\bar{Q}_{n}\left(\xi_{i}^{(n)}\right)\right| /\left|P_{n}^{\prime}\left(\xi_{i}^{(n)}\right)\right|$ are the weights of the $n$-point Gauss-Legendre rule, $h_{n}=2 /(2 n+1), k_{n}=(2 n) ! /\left(2^{n}(n !)^{2}\right)$ and $q_{n+1}(x)=1 /\left(2^{n} \lambda_{0}\right) E_{n+1}(x)$. Recalling (7), from (8) we have

$$
A_{i}^{(n)}>H_{i}^{(n)}\left(1-\frac{h_{n}}{k_{n}} 2^{n-1} \lambda_{0}\right)=0,
$$

which proves the theorem.

What follows is an immediate consequence (see for example [5, Theorem 15.2.2]) of the theorem we have just proved.

COROLlARY. The quadrature process defined by (1) is convergent for every function $f(x)$ which is Riemann-integrable in $[-1,1]$, i.e., $\lim _{n \rightarrow \infty} R_{n}(f)=0$.

Remark. In his paper, Szegö derives, although not explicitly stated, the analogue of (6) for rules of type (1) with a weight function of the form $\left(1-x^{2}\right)^{\mu-1 / 2}$, when 
$0<\mu<1$. In a way very similar to the Legendre case, it may then be shown that the weights of that type of rules are positive, too. For $\mu=0,1$ see [2].

Istituto di Calcoli Numerici

Università di Torino

10123 Torino, Italy

1. P. J. DAVIS \& P. RABINOWITZ, Methods of Numerical Integration, Academic Press, New York, 1975.

2. G. MONEGATO, "A note on extended Gaussian quadrature rules," Math. Comp., v. 30, 1976, pp. 812-817.

3. R. PIESSENS \& M. BRANDERS, "A note on optimal addition of abscissas to quadrature formulas of Gauss and Lobatto type," Math. Comp., v. 28, 1974, pp. 135-139

4. G. SZEGÖ, "Über gewisse orthogonale Polynome, die zu einer oszillierenden Belegungsfunktion gehören," Math. Ann., v. 110, 1934, pp. 501-513.

5. G. SZEGö, Orthogonal Polynomials, Amer. Math. Soc. Colloq. Publ., Vol. 23, 4th ed., Amer. Math. Soc., Providence, R. I., 1975. 